ARTICULO ORIGINAL

Rev Colombiana Cienc Anim 2017; 9(1)25-30.

\title{
Murciélagos asociados al campus de la Universidad de Córdoba, Montería, Colombia
}

\author{
Bats associated to the campus of the University of Cordoba, \\ Montería, Colombia
}

\author{
Chacón-Pacheco, Julio* Biol, Viloria-Rivas, José1 Biol, Ramos-Madera, Camilo Biol.
}

${ }^{1}$ Universidad de córdoba, Facultad de Ciencias Básicas, Grupo de Investigaciones Biodiversidad. Colombia Carrera 6 No. 76-103, Montería, Córdoba, Colombia.

\section{Keywords:}

Urban;

refuges;

trophic guilds;

Chiroptera

\section{Abstract}

The objective of this study was determined the richness, abundance and the use of refuges by bats associated to an urban area, the University of Cordoba in the Montería City, Colombia. Two methods were used, review of refuges from February 2011 to October 2015 and capture with mist nets of $6 \times 3 \mathrm{~m}$ between the months of August and November of 2014, and the months of May, June, August and October 2015, the nets were located between 18:00 and 24:00 hours. The methods complementarity allowed the registration of 113 individuals distribuited in ten species, nine genera and four families. The family Phyllostomidae and Molossidae were recorded by mist nets and revision of refuges, the families Emballonuridae and Noctilionidae only in the revision of refuges. For trophic guilds found greater representativity of guild frugivorous with four species, followed by insectivores (3), nectarivores (1), omnivores (1) and piscivores (1). This suggests that areas anthropic with structural complexity as the campus of the University of Córdoba allowed the maintenance of species associated with the supply of food and shelters.

\section{Resumen}

Se determinó la riqueza, abundancia y el uso de refugios por murciélagos asociados a un área urbana, la Universidad de Córdoba ubicada en la ciudad de Montería, departamento de Córdoba, Colombia. Se aplicaron dos métodos, revisión de refugios desde febrero de 2011 hasta octubre de 2015 y captura con redes de niebla de 6x3 m entre los meses de agosto y noviembre de 2014, y los meses de mayo, junio, agosto y octubre de 2015, las redes fueron ubicadas entre las 18:00 y 24:00 horas. La complementariedad de métodos permitió el registro de 113 individuos distribuidos en diez especies, nueve géneros y cuatro familias. Las familias Phyllostomidae y Molossidae fueron registradas por capturas en redes y revisión de refugios, mientras que las familias Emballonuridae y Noctilionidae solo en la revisión de refugios. Para los gremios tróficos se encontró mayor representatividad del gremio frugívoro con cuatro especies, seguido por tres insectívoros, un nectarívoro, un omnívoro y un piscívoro. Esto sugiere que zonas antropizados con complejidad estructural como el campus de la Universidad de Córdoba permiten el manteamiento de especies asociadas a la oferta de alimentos y refugios. 


\section{Introducción}

Los estudios sobre murciélagos han permitido comprender aspectos de su biología y su relación con los bosques tropicales y agroecosistemas (MEDELLíN, 2000; BALLESTEROS, 2015), sin embargo, para ambientes urbanos existen pocos análisis que permiten comprender como las especies logran adaptarse a estas condiciones, igualmente no se reconoce como se reparten y aprovechan los recursos (JUNG-K Y THRELFALL-C, 2015).

Para Colombia se reportan algunos estudios que documenten aspectos ecológicos de los murciélagos en ecosistemas urbanos (ALBERICO et al., 2005; SAMPEDRO et al., 2007; BALLESTEROS Y RACEROCASARRUBIA, 2012), existen estudios que relacionan los murciélagos y el virus rabia (OTERO et al., 1993; NÚÑEZ et al., 2001; BADILLO et al., 2009; NÚÑEZ et al., 2012; BRITO-HOYOS et al., 2013; RANGEL et al., 2014).

De este mismo modo, al noreste del país, en el departamento de Córdoba, dado el desarrollo de prácticas agrícolas se ha llegado a la pérdida de la cobertura original de los bosques tropicales (CALONGE et al., 2010), por lo cual, la mayoría de estudios se orientan en conocer sobre la ecología de murciélagos en los últimos remanentes de bosques y en sistemas agrícolas y ganaderos (BALLESTEROS y RACERO-CASARRUBIA, 2012; CHACÓN et al., 2015a; BALLESTEROS, 2015). A pesar del creciente aumento de la población humana en el Departamento, que promueve la ampliación de las fronteras urbanas, los trabajos sobre el conocimiento de los murciélagos inmersos en estos ambientes han sido escasos, se han realizado los trabajos de SUAREZ-VILLOTA et al. (2009) y BALLESTEROS y RACERO-CASARRUBIA (2012), donde no se han evaluado el uso de refugios, lo cual podría ser importante para emprender programas de conservación y resolver los conflictos creados con ellos por su presencia en las ciudades (ALBERICO et al., 2005).

De acuerdo a lo anterior y a la falta de información sobre la relación entre los murciélagos y los ambientes urbanos para el Departamento y en Colombia, este trabajo tiene como objetivo conocer la diversidad y el uso del espacio (refugios) de las especies dentro de ambientes urbanizados como es el caso del campus de la Universidad de Córdoba.

\section{Materiales y Métodos}

Área de estudio. El estudio se llevó a cabo en el campus de la Universidad de Córdoba ubicada en la ciudad de Montería, departamento de Córdoba, Colombia $\left(8^{\circ} 47^{\prime} 26,5^{\prime \prime} \mathrm{N} 75^{\circ} 51^{\prime} 39,6^{\prime \prime} \mathrm{W}\right)$. En la zona predomina un régimen de precipitaciones bimodal con un promedio anual de $1300 \mathrm{~mm}$, una temperatura promedio de $28^{\circ} \mathrm{C}$ y 5,42 horas diarias de brillo solar (IGAC, 1996; PALENCIA-SEVERICHE et al., 2006). La Universidad presenta un área de 57 ha que limitan con el Río Sinú, se encuentra ocupada en su mayoría por zonas agrícolas, piscícolas, inmuebles (edificaciones, aulas y cubículos de un solo piso), zonas verdes y fragmentos de vegetación riparia (Figura 1). La Universidad se caracteriza por presentar especies frutales de plantas nativas como higos (Ficus spp), zapote (Manilkara zapota), caimito (Pouteria caimito), guayaba (Psidium guajava), guayaba agría (Psidium. Araca), jobo (Spondias mombin), tamarindo (Tamarindus indica), entre otras y algunas introducidas como mango (Mangifera indica) y pera de agua (Syzygium malaccense).

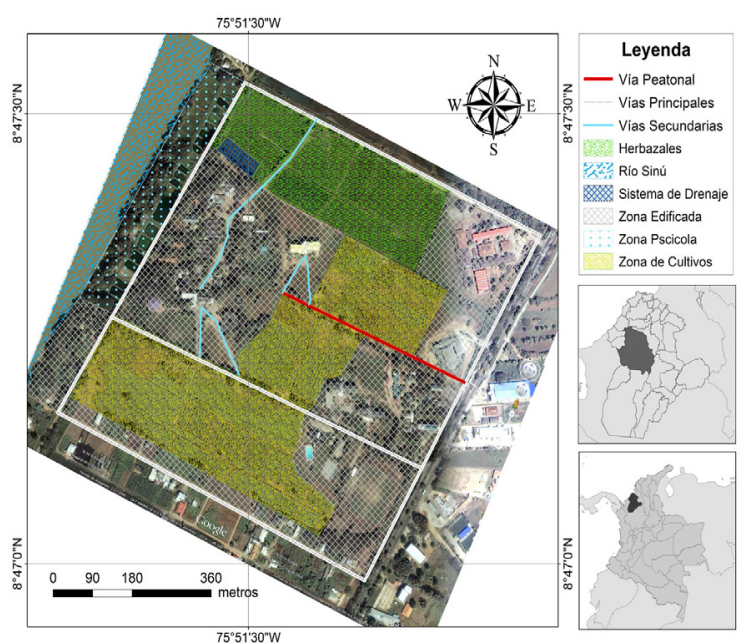

Figura 1. Localización y descripción de la Universidad de Córdoba, Montería - Córdoba, Colombia.

Métodos. En este trabajo se aplicaron dos métodos de estudio, el primero consistió en la revisión aleatoria de posibles refugios desde el año 2011 hasta octubre de 2015 y captura de especímenes con ayuda de una jama. En segundo lugar, se utilizaron cuatro redes de niebla de $6 \times 3 \mathrm{~m}$ entre las 18:00 hasta las 00:00 horas para la captura de murciélagos entre los meses de agosto y noviembre de 2014, y los meses entre marzo a junio, agosto y octubre de 2015, para un total de 16 noches efectivas de muestreo. Las redes se revisaron cada 30 minutos para la colecta de los especímenes capturados. Los murciélagos fueron depositados en bolsas de tela y se transportaron al lugar de procesamiento. Se colectó en lo posible un espécimen de cada sexo para cada especie, los cuales fueron depositados en la Colección Zoológica de la Universidad de Córdoba (CZUC). 
Para la identificación se emplearon las claves taxonómicas de EMMONS y FEER (1997), TIM y LAVAL (1998) y LINARES (1998), además de las descripciones de GARDNER (2008). Cada especie capturada se ubicó en un gremio trófico según la clasificación de KALKO (1997) y SORIANO (2000).

\section{Resultados}

Se registraron 113 individuos pertenecientes a diez especies, nueve géneros y cuatro familias. Con el uso de redes de niebla se capturaron 85 individuos de seis especies, con un esfuerzo de captura de $384 \mathrm{~h} / \mathrm{red}$ y un éxito de captura de 0,221 individuos/horas-red. Para la revisión de refugios se registraron 28 individuos de siete especies. Se encontró que Artibeus lituratus (25,7\%), A. planirostris $(24,8 \%)$ y Glossophaga soricina $(14,2 \%)$ fueron las especies más abundantes. En cuanto a los gremios tróficos, las especies frugívoras presentaron mayor representatividad $(55,8 \%)$ de los individuos capturados, seguido de los insectívoros $(22,1 \%)$, nectarívoros $(14,2 \%)$, omnívoros $(4,4 \%)$ y piscívoros $(3,5 \%)$ (Tabla 1).

Los refugios registrados en la Universidad de Córdoba corresponden a árboles de las especies Ficus sp, Mangifera indica y Sterculia apetala, tejados, aberturas en cielo raso y grietas en las edificaciones. La familia Phyllostomidae y Molossidae fueron registradas por capturas en redes y revisión de refugios, mientras que las familias Emballonuridae y Noctilionidae solo en la revisión de refugios. En los tejados se registraron tres especies, seguido de las plantas Ficus sp y $S$. apetala, y el cielo raso con dos especies para cada tipo de refugio. Las especies más generalistas en cuanto al uso de hábitat (refugios) fueron Artibeus lituratus y Molossus molossus, encontrándose en tres de los refugios reportados (Tabla 2 ).

Tabla 1. Listado de especies de murciélagos presentes en la Universidad de Córdoba.

\begin{tabular}{|c|c|c|c|c|}
\hline Familia & Especie & Gremio & Refugios & Redes \\
\hline \multirow[t]{6}{*}{ Phyllostomidae } & $\begin{array}{l}\text { Artibeus lituratus } \\
\text { (Olfers, 1818) }\end{array}$ & FR & 1 & 28 \\
\hline & $\begin{array}{l}\text { Artibeus planirostris } \\
\quad \text { (Spix, 1823) }\end{array}$ & FR & 3 & 25 \\
\hline & $\begin{array}{c}\text { Sturnira lilium } \\
\text { (É. Geoffroy, 1810) }\end{array}$ & FR & & 5 \\
\hline & $\begin{array}{l}\text { Uderma convexum } \\
\quad \text { (Lyon 1902) }\end{array}$ & FR & 1 & \\
\hline & $\begin{array}{c}\text { Glossophaga soricina } \\
\text { (Pallas, 1766) }\end{array}$ & NE & & 16 \\
\hline & $\begin{array}{l}\text { Phyllostomus discolor } \\
\text { (Wagner, 1843) }\end{array}$ & OM & & 5 \\
\hline Molossidae & $\begin{array}{l}\text { Molossus molossus } \\
\quad(\text { Pallas, 1766) }\end{array}$ & IN & 8 & 6 \\
\hline \multirow[t]{2}{*}{ Emballonuridae } & $\begin{array}{l}\text { Rhynchonycteris naso } \\
\text { (Wied-Neuwied, 1820) }\end{array}$ & IN & 6 & \\
\hline & $\begin{array}{l}\text { Saccopteryx leptura } \\
\text { (Schreber, 1774) }\end{array}$ & IN & 5 & \\
\hline Noctilionidae & $\begin{array}{l}\text { Noctilio albiventris } \\
\text { (Desmarest, 1818) }\end{array}$ & $\mathrm{PI}$ & 4 & \\
\hline & Total & & 28 & 85 \\
\hline
\end{tabular}

FR: Frugívoro; IN: Insectívoro; NE: Nectarívoro; OM: Omnívoro; PI: Piscívoro.

Tabla 2. Murciélagos registrados por tipo de refugios en la Universidad de Córdoba.

\begin{tabular}{|c|c|c|c|c|c|c|}
\hline \multirow{3}{*}{ Especies } & \multicolumn{6}{|c|}{ Refugios } \\
\hline & \multicolumn{3}{|c|}{ Naturales } & \multicolumn{3}{|c|}{ Artificiales } \\
\hline & Mangifera indica & Ficus sp & Sterculia apetala & Cielo Raso & Tejado & Grietas \\
\hline Rhynchonycteris naso & & & & & 6 & \\
\hline Saccopteryx leptura & 3 & & & & 2 & \\
\hline Noctilio albiventris & & & 4 & & & \\
\hline Molossus molossus & & & 6 & 1 & & 2 \\
\hline Uroderma billobatum & & & & & 1 & \\
\hline Artibeus lituratus & & 1 & & 1 & & \\
\hline Artibeus planirostris & & 1 & & & & \\
\hline Total & 3 & 2 & 10 & 2 & 9 & 2 \\
\hline
\end{tabular}




\section{Discusión}

Las especies de murciélagos capturados en la Universidad de Córdoba corresponden al 18,86\% de las especies mencionadas para el departamento de Córdoba (RACERO-CASARRUBIA et al., 2015). A pesar de la baja representatividad de especies, fue posible el registro de cuatro de las nueve familias reportadas para Córdoba y Colombia (SOLARI et al., 2013). No se descarta la presencia de otras especies que podrían no haberse registrado por los métodos utilizados, de acuerdo a esto, se reconoce que el uso de métodos complementarios como la revisión de refugios permitieron para este trabajo el registro de especies de baja capturabilidad con el uso de redes de niebla, por lo cual se confirma que el uso de distintos métodos permiten lograr una caracterización más completa y precisa de la quiropterofauna (MORA et al., 2002; LÓPEZ-BERRIZBEITIA y DIAZ, 2013).

A pesar de la baja disponibilidad de refugios naturales en el campus de la Universidad, los refugios artificiales permiten la acomodación de los murciélagos, como se ha reportado en otros estudios donde las edificaciones brindan alternativas para las especies, cumpliendo con los requisitos de espacio, humedad y temperatura para albergar colonias de murciélagos caseros (ALBERICO et al., 2005), es así, que los refugios artificiales, como los tejados, permitieron la presencia de tres especies, incluyendo a Rhynchonycterix naso y Saccopteryx leptura, las cuales son indicadas en otros estudios como especies raras, con bajas densidades de captura (SUÁREZ-VILLOTA et al., 2009; VELA-VARGAS y PÉREZ-TORRES, 2012; CHACÓN et al., 2015a).

En cuanto al análisis por familias, se encontró que Phyllostomidae fue predominante, la cual es considerada de gran adaptabilidad a ecosistemas con alto grado de intervención humana, gracias a sus particularidades fisiológicas y conductuales, que le permiten aprovechar una variedad de recursos disponibles en el medio (BALLESTEROS y LINARES, 2015). Así mismo, dentro de esta familia fue posible la captura principalmente de especies frugívoras, las cuales tuvieron la mayor representatividad de individuos, debido a la disponibilidad de árboles frutales, y la incidencia con sus periodos de floración y fructificación que podrían estar representando una oferta importante de alimento.

Por otro lado, la variedad de ambientes dentro del campus universitario promueve la presencia de diversos grupos de murciélagos, así como las iluminaciones y cultivos presentes que sirven como hábitat permanente de muchas especies de insectos, sirviendo a su vez de soporte energético a los murciélagos insectívoros, los cuales se han anotado como abundantes en zonas urbanas (ALBERICO et al., 2005). Del mismo modo, la ubicación cercana del Rio Sinú y el establecimiento de un centro de investigación piscicola dentro la Universidad de Córdoba, sirven a su vez para el mantenimiento de especies piscívoras como $N$. albiventris y de especies omnívoras como Phyllostomus discolor.

Estos resultados y los expuestos por (CHACÓN et al., $2015 b$ ) indican que zonas antropizadas con complejidad estructural como el campus de la Universidad de Córdoba permiten el manteamiento de especies silvestres asociados a la oferta de alimentos y refugios.

Agradecimientos: A Richard Hernández y Daniela Mena por facilitar el shapefile de la Universidad de Córdoba. A Gina Reyes Castellar por la revisión del abstract.

\section{Referencias}

ALBERICO, M.; SAAVEDRA, C.; GARCÍA-PAREDES, H. 2005. Murciélagos caseros de Cali (Valle del caucaColombia). Caldasia $27(1): 117-126$.

BADILLO, R.; MANTILLA, J.; PRADILLA, G. 2009. Encefalitis rábica humana por mordedura de murciélago en un área urbana de Colombia. Biomédica 29 (2):191-203.

BALLESTEROS, J.; RACERO-CASARRUBIA, J. 2012. Urban Bats from the City of Montería, Córdoba-Colombia. Revista MVZ Córdoba 17 (3):3193-3199.

BALLESTEROS, J.; LINARES, J. 2015. Fauna de Córdoba, Colombia. Grupo Investigación Biodiversidad UniCórdoba, Facultad de Ciencias Básicas. Fondo Editorial, Universidad de Córdoba. Bogotá.

BALLESTEROS, J. 2015. Efecto del manejo silvopastoril y convencional de ganadería extensiva sobre el ensamblaje de murciélagos asociados a fragmentos de bosque seco tropical en Córdoba, Colombia. Tesis Doctoral. Facultad de Ciencias, Pontificia Universidad Javeriana, Bogotá. 
BRITO-HOYOS, D.; SIERRA, E.; ÁLVAREZ, R. 2013. Distribución geográfica del riesgo de rabia de origen silvestre y evaluación de los factores asociados con su incidencia en Colombia, 1982-2010. Revista Panamericana de Salud Pública 33 (1):8-14.

CALONGE, B.; VELA-VARGAS, I.; PÉREZ-TORRES, J. 2010. Murciélagos asociados a una finca ganadera en Córdoba (Colombia). Revista MVZ Córdoba 15 (1):1938-1943.

CHACÓN, J.; HUMANEZ, E.; MARTÍNEZ, J. 2015a. Murciélagos asociados con un área de restauración ecológica en el Alto Sinú, departamento de Córdoba, Colombia. Revista Biodiversidad Neotropical 5 (2):120-123.

CHACÓN, J.; HUMANEZ, E.; ESPITIA-CARRILLO, Y. 2015b. Carnívoros en la Universidad de Córdoba, Colombia: Análisis de la Relación Fauna Silvestre-Comunidad Urbana. Notas Mastozoológicas 2 (1):44-46.

EMMONS, L.; FEER, F. 1997. Neotropical rainforest mammals: A field guide. 2nd ed. The University of Chicago Press. Chicago.

GARDNER, A. L. 2008. Mammals of South America, Volume 1. Marsupials, Xenarthrans, Shrews and Bats. The University of Chicago Press. Chicago.

INSTITUTO GEOGRÁFICO AGUSTÍN CODAZZI (IGAC). 1996. Diccionario geográfico de Colombia. Tomo 3. IGAC Ediciones. Bogotá.

JUNG, K.; THRELFALL, C. 2016. Urbanisation and Its Effects on Bats-A Global Urbanisation and Its Effects on Bats-A Global Meta-Analysis. Pags. 13-33. En: Voigt, C.; Kingston, T. (eds), Bats in the Anthropocene: Conservation of Bats in a Changing World. Springer International Publishing AG. London.

KALKO, E. 1997. Diversity in tropical bats. Pags. 13-43. En: Ulrich, H. (ed.). Tropical biodiversity and systematics. Zoologisches Forschungsinstitut und Museum Alexander Koenig. Bonn, Alemania.

LINARES, O. 1998. Mamíferos de Venezuela. Sociedad Conservacionista Audobun de Venezuela. Caracas.

LÓPEZ-BERRIZBEITIA, M.; DÍAZ, M. 2013. Diversidad de murciélagos (Mammalia, Chiroptera) en la ciudad de Lules, Tucumán. Acta Zoológica Mexicana 29 (1):234-239.

MEDELLÍN, R.; EQUIHUA, M.; AMIN, M. 2000. Bat diversity and abundance as indicators of disturbance in Neotropical rainforests. Conservation Biology 14 (6):1666-1675.

MORA, E.; MACÍAS, S.; ROJAS, D.; RODRÍGUEZ, A.; QUIÑONEZ, I.; GARCÍA, A.; CÁDIZ, A.; BOBURG, B. 2002. Aplicación de métodos bioacústicos y convencionales en la caracterización de la comunidad de murciélagos de la Cueva del Indio, Tapaste, la Habana, Cuba. Revista Biología 16 (2):159-166.

NÚÑEZ, M.; ALDAZ, J.; ESCOBAR, H.; CUADROS, M. 2001. Primer hallazgo de rabia silvestre en el murciélago insectívoro Eptesicus brasiliensis en Cali, Colombia. Revista de la Asociación Colombiana de Ciencias Biológicas 12:57-61.

NÚÑEZ, C.; PÁEZ, A.; HERNÁNDEZ, C.; ESCOBAR, H.; BONELO, A. 2012. Transmisión del virus de la rabia entre murciélagos urbanos del departamento del Valle del Cauca, Colombia, 1999-2008. Infectio 16 (1):23-29.

OTERO, J.A.; ARANGO-JARAMILLO, S.; RESTREPO, M.T.; ALBERICO, M.; GUTIÉRREZ, H.; MÁRQUEZ, M.; OLIVEROS, A.; ESCOBAR, H.; LOSADA, E.; SARDI, R.; VALENCIA, D.; SARDI, H.; BUITRAGO, L. 1993. Prevalencia de virus rábico en quirópteros del área municipal de Cali, Colombia. Colombia Médica 24:137-141.

PALENCIA-SEVERICHE, G.; MERCADO-F, T.; COMBATH-CABALLERO, E. 2006. Estudio agroclimático de Córdoba. Facultad de Ciencias Agrícolas. Universidad de Córdoba. Montería, Colombia.

RACERO-CASARRUBiA, J.; BALLESTEROS, J., PEREZ-TORRES, J. 2015. Mamíferos del departamento de Córdoba-Colombia: historia y estado de conservación. Biota Colombiana 16 (2):128-148. 
RANGEL, A.; RODRÍGUEZ, V.; TERAN, C. 2014. Análisis filogenético del virus de la rabia en mamíferos reportados en Colombia entre 1994 y 2008. Revista Ciencia y Agricultura 11 (2):69-75.

SAMPEDRO, A.; MARTINEZ, C.; DE LA OSSA, K.; OTERO, Y.; SANTOS, L.; OSORIO, S.; MERCADO, A. 2007. Nuevos registros de especies de murciélagos para el departamento de Sucre y algunos datos sobre su ecología en esta región colombiana. Caldasia 29 (2):355-362.

SOLARI, S.; MUÑOZ-SABA, Y.; RODRÍGUEZ-MAHECHA, J.; DEFLER, T.; RAMÍREZ-CHAVES, H.; TRUJILLO, F. 2013. Riqueza, endemismo y conservación de los mamíferos de Colombia. Mastozoología neotropical 20 (2):301-365.

SORIANO, P. 2000. Functional structure of bat communities in tropical rainforest and Andean Cloud Forest. Ecotropicos $13(1): 1-20$.

SUÁREZ-VILLOTA, E.; RACERO-CASARRUBIA, J.; GUEVARA, G.; BALLESTEROS, J. 2009. Evaluación ecológica rápida de los quirópteros del parque ecológico de Montelíbano, Córdoba, Colombia. Tropical Conservation Science $2(4): 437-449$.

TIMM, R.; LAVAL, R. 1998. A fiel key to the bats of Costa Rica. Occasional Publication Series University of Kansas Center of Latin American Studies 22:1-30.

VELA-VARGAS, I.; PÉREZ-TORRES, J. 2012. Murciélagos asociados a remanentes de bosque seco tropical en un sistema de ganadería extensiva (Colombia). Chiroptera Neotropical 18 (1):1089-100. 